\title{
Médiévales
}

Langues, Textes, Histoire

53 | automne 2007

La nature en partage

\section{Des « prouffitz champestres » à la gestion des ressources naturelles}

\section{Philippe Bernardi et Didier Boisseuil}

\section{(2) OpenEdition}

1 Journals

Édition électronique

URL : https://journals.openedition.org/medievales/3173

DOI : $10.4000 /$ medievales. 3173

ISSN : $1777-5892$

Éditeur

Presses universitaires de Vincennes

\section{Édition imprimée}

Date de publication : 1 décembre 2007

Pagination : 5-10

ISBN : 978-2-84292-211-5

ISSN : 0751-2708

Référence électronique

Philippe Bernardi et Didier Boisseuil, « Des « prouffitz champestres » à la gestion des ressources naturelles », Médiévales [En ligne], 53 | automne 2007, mis en ligne le 17 décembre 2009, consulté le 22 avril 2022. URL : http://journals.openedition.org/medievales/3173 ; DOI : https://doi.org/10.4000/ medievales.3173 
Philippe BERNARDI

Didier BOISSEUIL

\section{DES « PROUFFITZ CHAMPESTRES » À LA GESTION DES RESSOURCES NATURELLES*}

Avant d'envisager l'étude des «ressources naturelles » exploitées au Moyen Âge ou leurs modes de mise en valeur, il n'est sans doute pas inutile de s'interroger sur la notion même de « ressources naturelles » ${ }^{1}$. Cette dernière paraît a priori bien entendue, servie qu'elle est par une expression dont la simplicité ou l'évidence laissent à penser que son sens commun peut se satisfaire d'une lecture littérale.

À bien y regarder, nous constatons néanmoins que seuls les dictionnaires $\mathrm{du} \mathrm{Xx}^{\mathrm{e}}$ siècle en font clairement mention. L'usage du terme polysémique de « ressources » apparaît en français, avec le sens de «moyens matériels dont dispose ou peut disposer une collectivité ${ }^{2}$ », à la fin du XVIII ${ }^{\mathrm{e}}$ siècle seulement. Et, si la notion de ressources naturelles émerge - disons - avec le début de la révolution industrielle ${ }^{3}$, la locution ne se trouve employée dans des titres d'ouvrages ou de mémoires en français qu'à partir de la fin du siècle suivant ${ }^{4}$. Son usage se développe dans les années 1920-1930 et connaît

* L'organisation de ce numéro a bénéficié du soutien financier du Laboratoire d'histoire «territoires, pouvoirs, identités » de l'université d'Avignon, du LAMM (UMR 6572) et du CIHAM (UMR 5648).

1. Elle fait suite à la notion de «richesses naturelles », de trésors, qui a cours à l'époque moderne et qui est à mettre en liaison avec les développements de l'économie politique, $c f$. A. COOPER, " "The Possibilities of the Land": The Inventory of "Natural Riches" in the Early Moderne German Territories » dans M. Schabas et N. De Marchi éd., Oeconomies in the Age of Newton, Londres, 2003, p. 131-153. Ces thèmes ont fait l'objet de plusieurs séminaires d'Alice Ingold, Administrer les « ressources naturelles » XIX ${ }^{e}-X X^{e}$ siècles, de l'École des Hautes Études en Sciences Sociales, lors de l'année 2004-2005.

2. Le grand Robert de la langue française, Paris, 1990 ( $2^{\mathrm{e}}$ éd.), 9 vol.

3. S. Gніотті, «Ressources », dans Dictionnaire des notions, Paris, 2005, p. 1023-1024.

4. Les plus anciens ouvrages en langue française que nous avons pu retrouver dont le titre ou le sous-titre comportent cette expression sont ceux d'Ernest Jean Van BRUYSSEL ( $\mathrm{La}$ république Argentine, ses ressources naturelles, ses colonies agricoles, son importance comme 
surtout une large diffusion vers le milieu du siècle, à travers, notamment, son adoption par des institutions internationales comme l'ONU qui fonde, en 1948, «l'Union internationale pour la conservation de la nature et des ressources naturelles »(UICN).

Il semble que les auteurs de langue française aient emboîté le pas de leurs collègues anglo-saxons en adoptant l'équivalent exact de la locution anglaise natural resources. C'est du moins ce qu'invite à penser le décalage observable entre les deux langues puisque, dès 1807, Joseph Corry publiait à Londres, une monographie sur les côtes africaines dont le titre annonce une étude Upon the Natural and Commercial Resources of the Country ${ }^{5}$.

Au-delà des questions linguistiques, il faut souligner que c'est surtout à travers une géographie attachée alors à la «connaissance rationnelle des ressources ${ }^{6}$ à des fins pour partie prospectives que la notion paraît, dans un premier temps, se diffuser. Vers les années 1950, les économistes y introduisirent des nuances, distinguant entre des ressources naturelles renouvelables et d'autres, non renouvelables, dont la quantité est donnée une fois pour toutes dans la nature. L'idée de gestion des ressources naturelles vint alors en corollaire de celle d'exploitation des ressources naturelles. La prise de conscience progressive de l'épuisement de ressources naturelles majeures (eau, pétrole, etc.) contribua à donner, depuis, à cette notion une dimension écologique importante en l'associant de plus en plus aux questions de pollution, d'économie d'énergie, de protection de l'environnement.

Ces dernières années, l'expression a ainsi vu sa définition s'enrichir de diverses nuances allant de l'idée, chère aux économistes, de « facteur de production originel, objet du travail humain et comprenant l'espace, les matières premières et l'énergie ${ }^{7}$ » pour désigner, du point de vue écologique, «l'ensemble des produits naturels, des milieux, des sites, des équilibres et de leurs modes traditionnels de gestion, dans la mesure où les éléments de cet ensemble sont menacés de disparition ou d'amoindrissement par le développement de la civilisation industrielle ${ }^{8} \gg$. Elle en vient même pour certains à être considérée comme « la productivité d'un lieu, ou biotope, par le seul jeu des facteurs

centre d'immigration, Bruxelles, 1888 et La république du Paraguay : découverte et colonisation du pays, son histoire, le territoire paraguayen, climat, ressources naturelles, population, voies de communication, agriculture, industrie pastorale et manufacturière, relations commerciales, situation financière, observations générales, Paris, 1893) ou de Rafael de ZAYAS ENRIQUEZ, Les États-Unis mexicains. Leurs ressources naturelles. Leurs progrès. Leur situation actuelle, Mexico, 1899.

5. J. CORRY, Observations upon the windward coast of Africa, the religion, character, customs, \& c., of the natives, with a system upon which they may be civilised, and a knowledge attained of the interior of this extraordinary quarter of the globe, and upon the natural and commercial resources of the country, Londres, 1807. À ce titre, il n'est peut-être pas sans intérêt de noter que les plus anciennes publications retrouvées portant l'expression « ressources naturelles » dans leur titre traitent de pays du continent américain.

6. S. GHIOTTI, loc. cit.

7. A. Silem et J.-M. Albertini, Lexique. Économie, Paris, 1995 (5 éd.).

8. Grand Larousse Universel, Paris, 1985, 18 vol. 
naturels et au premier chef l'activité des biocénoses animales et végétales qui le peuplent et composent l'écosystème local ${ }^{9}$ ».

Si la locution a bel et bien une histoire, sa définition, loin de connaître une évolution linéaire, est allée se complexifiant par l'accumulation de variantes. Elle désigne - hormis selon les définitions écologiques les plus poussées - les éléments du monde bio-physique utilisés par les sociétés humaines et plus étroitement les sociétés occidentales industrialisées. Elle témoigne d'une conception de la Nature propre à l'Occident ${ }^{10}$, qui prend forme justement au Moyen Âge. En effet, la pensée scolastique des XII et XIII ${ }^{\mathrm{e}}$ siècles impose l'idée d'une Nature destinée à l'homme, créée par Dieu et « indépendante du drame humain ${ }^{11}$ ».

Les ouvrages à caractère encyclopédique des XIII ${ }^{\mathrm{e}}-\mathrm{XIV}^{\mathrm{e}}$ siècles, empreints de cette doctrine, témoignent, à travers les classifications savantes qu'ils établissent et les descriptions qu'ils proposent, d'une attention à ce que nous pouvons reconnaître comme des ressources naturelles. Cela transparaît plus nettement encore dans les traités d'agronomie, aux finalités économiques affirmées ${ }^{12}$. Dans son Liber ruralium Commodorum (1304-1309), Piero de' Crescenzi - suivant pour partie quelques auteurs antiques comme Palladius ou Columelle - qualifie les terres et les sources d'eau les plus profitables à la croissance des plantes et aux besoins des hommes. Le caractère utilitaire de son entreprise ressort d'ailleurs plus aisément dans le titre de la traduction française de son ouvrage, réalisée à la fin du XIV ${ }^{\mathrm{e}}$ siècle : Le livre des prouffitz champestres et ruraulx. Nous retrouvons, enfin, les mêmes préoccupations dans les traités d'architecture de la fin du Moyen Âge. Se détachant de considérations générales sur les qualités des divers matériaux qu'il emprunte essentiellement à Vitruve, l'ingénieur siennois Francesco di Giorgio Martini porte, par exemple, dans l'un des ses traités ${ }^{13}$, un regard aigu sur les ressources

9. F. RAMADE, «Ressources naturelles», dans Dictionnaire des notions, Paris, 2005, p. 1024-1026.

10. C'est ce que soulignent les anthropologues contemporains - tout particulièrement Philippe Descola dans un ouvrage récent Par-delà nature et culture, Paris, 2005. Une bonne présentation des enjeux de la conception de la Nature pour la sociologie contemporaine se trouve dans Chassez le naturel... écologisme, naturalisme et constructivisme. Revue du MAUSS, 17, 2001.

11. R. Lenoble, «L'héritage de l'Antiquité et du Moyen Âge. Le monde de Lucrèce et le monde de Dante pour les hommes du XVI siècle » dans J. BEAudE éd., Histoire de l'idée de nature, Paris, 1969, p. $261 s q$. ; voir aussi T. GREgORY, « Nature» dans J. Le Goff, J.-C. SchmitT éd., Dictionnaire raisonné de l'Occident médiéval, Paris, 1999, p. 806-819; Ph. Descola, « L'anthropologie et la question de la nature» dans M. Abélès, L. Charles, H. P. Jendy, B. Kalaora éd., L'environnement en perspective. Contexte et représentation de l'environnement, Paris, 2000, p. 61-84, p. 61 ; quelques éléments repris dans C. et R. LARRÉRE, Du bon usage de la nature. Pour une philosophie de l'environnement, Paris, 1997.

12. L'agronomie traite, en effet, d'un aspect de l'économie : la gestion du domaine rural, cf. J.-L. Gaulin, Pietro de' Crescenzi et l'agronomie en Italie (XII ${ }^{e}-X I V^{e}$ s.), thèse de doctorat dir. P. Toubert, Université de Paris I, 1990.

13. Francesco di Giorgio MarTINI, Tratatti di architettura, ingegneria et arte militare, éd. C. Maltese, transcrip. L. Maltese Degrassi, Milan, 1967, t. 2, p. 309-311. 
de l'espace toscan : au côté des marbres de Paros, il énumère ceux de Carrare, de Cerbaia (tout près de Sienne dans la Montagnola), le marbre rouge de Gerfalco, le porphyre de l'Albarese, etc.

Historiens et archéologues ont étudié et étudient précisément la façon dont les hommes du Moyen Âge se sont emparés de certaines de ces ressources. En attestent, par exemple, les recherches sur les mines et la métallurgie médiévales, les enquêtes sur les carrières et les forêts, les travaux sur les plantes tinctoriales, les études environnementales ${ }^{14}$ ou les travaux sur l'histoire des droits d'usage, menés notamment par les juristes (les usi civici) ${ }^{15}$. Toutefois, la notion de ressources naturelles (dont les historiens de l'Antiquité commencent à faire usage ${ }^{16}$ ) est encore peu utilisée par les médiévistes ${ }^{17}$. L'anachronisme de l'expression ne doit cependant pas nous conduire à nous priver d'une notion qui peut permettre d'explorer les conditions matérielles de l'existence sociale de l'homme médiéval à travers l'étude des modes d'appropriation de la nature ${ }^{18}$. Du fait même de sa complexité, cette notion présente un intérêt pour les historiens puisqu'elle permet d'articuler des enjeux économiques, techniques, politiques et environnementaux et, partant, d'offrir une vision plus fine de la société considérée. Encore faut-il, pour cela, s'entendre sur ce que l'on désigne par « ressources naturelles».

Aussi, avons-nous proposé de retenir comme «ressources naturelles » - parce que cela nous semblait plus proche des définitions évoquées précédemment - l'ensemble des éléments de la nature (au sens de monde biophysique) qui entrent dans un processus de production artisanale ou industrielle, et qui ne sont pas cultivés ou élevés par l'homme; ce qui exclut toutes les productions agricoles et les ressources alimentaires.

$\mathrm{Au}$ vu de cette définition, nous retiendrons des objets animés ou inanimés : les ressources minières (au sens large, de tous les minéraux, pas seulement les minerais métalliques), les pierres (qu'elles soient précieuses ou ordinaires),

14. Dont un exemple, Burnouf J., Leveau Ph. dir., Fleuves et marais au croisement de la nature et de la culture, Paris, 2004.

15. Une approche récente, M. BouRIN, «Les droits d'usage et la gestion de l'inculte en France méridionale : un terrain de comparaison "avant la Peste noire" » dans BouRIN M., BoIsSELIER S. dir., L'espace rural au Moyen Âge. Portugal, Espagne, France (XII ${ }^{e} X I V^{e}$ s.). Mélanges en l'honneur de Robert Durand, Rennes, 2002, p. 193-206. L'étude des usi civici fut ces dernières années menée en partie dans le "Centro di Studi e documentazione sui domani civici e le proprietà collettive » de l'università di Trento.

16. M. Clavel-LÉVÊQue, E. HeRmon éd., Espaces intégrés et ressources naturelles dans l'Empire romain, Besançon, 2004.

17. Hormis la récente publication de A. CATAFAU éd., Les ressources naturelles des Pyrénées du Moyen Âge à l'époque moderne, exploitation, gestion, appropriation, Perpignan, 2005.

18. "Si l'on admet l'hypothèse de Marx qui veut que les rapports sociaux dominent le fonctionnement d'ensemble de la production d'une société parce qu'ils fonctionnent comme des rapports de production, alors les rapports sociaux qui servent de cadre à l'appropriation matérielle de la nature et donc produisent les conditions matérielles de l'existence sociale jouent un rôle déterminant », M. Godelier, L'idéel et le matériel. Pensée, économies, sociétés, Paris, 1984, p. 33. 
les terres (pour tous les usages : entre autres la céramique), les ressources hydrauliques (les cours d'eau utilisés pour leurs capacités énergétiques, curatives, d'irrigation), les ressources forestières (pour les combustibles ou le bois d'œuvre qu'elles produisent) et plus largement les plantes (les plantes tinctoriales notamment, les fibres textiles lorsqu'elles ne sont pas issues de la culture) ; les ressources animales (comme les cochenilles du chêne kermès), les ressources maritimes (sel), etc.

Cette notion de « ressources naturelles » implique un ensemble d'opérations sociales qui - selon une approche anthropologique ${ }^{19}$ - relèvent de la prédation ou de l'exploitation (c'est-à-dire de l'utilisation raisonnée des ressources qui passe par la cueillette, la captation, par opposition à la mise en culture) : cela entraîne tout d'abord une sélection. En sorte que les « ressources naturelles » sont des ressources inventées et non données. Elles n'existent qu'en raison d'une demande sociale et il n'est pas possible de considérer la nature comme un simple stock. Leur présence n'est pas nécessairement illimitée, en sorte que la notion de « ressources naturelles » peut impliquer l'idée de réserve ou de gisement.

Ensuite cette définition induit l'étude du conditionnement qui rend les ressources utiles à un usage productif ultérieur. Les ressources ne sont pas en effet nécessairement des matières premières, car elles sont en amont dans la chaîne de production. Le bois, la pierre nécessitent, par exemple, une préparation avant d'être utilisés pour la construction. Cette précision impose d'étudier les techniques, les procédés, les instruments et l'organisation du travail qui permettent de semblables transformations ${ }^{20}$.

Cette approche amène également à réfléchir sur les processus d'appropriation: les modalités - matérielles, symboliques - par lesquelles les hommes manifestent leur prise de possession des ressources. Elle conduit, enfin, à considérer l'espace où sont exploitées les ressources et donc la définition, l'étude d'un territoire ${ }^{21}$.

19. Qui rejoint celle formulée par C. BeCK, Y. LuginBÜHL, « Sociétés et ressources renouvelables. Temps et espaces des crises environnementales », Natures, Sciences, Sociétés, 9-3, 2001, p. 74-78.

20. «Une réalité naturelle devient une ressource pour l'homme par l'effet combiné de deux conditions : qu'elle puisse directement ou indirectement satisfaire un besoin humain (posséder une utilité au sein d'une forme de vie sociale); que l'homme dispose de moyens techniques de la séparer du reste de la nature et la faire servir à ses fins », M. GodeliER, L'idéel et le matériel, op. cit., p. 112. Sur le rôle des techniques dans les processus de conditionnement des ressources naturelles, $c f$. P. BECK dir., L'innovation technique au Moyen Âge, Actes du VI ${ }^{e}$ Congrès International d'archéologie médiévale, Paris, 1998 ; L. Hilaire-Pérez, C. Verna, « Dissemination of Technical Knowledge in the Middle Ages and the Early Modern Era », Technology and Culture, 47, 2006, p. 536-565.

21. Il s'agit d'«une portion de l'espace sur lequel une société déterminée revendique et garantit à tout ou une partie de ses membres des droits stables d'accès, de contrôle et d'usage portant sur tout ou partie des ressources qui s'y trouvent et qu'elle est désireuse et capable d'exploiter », M. Godelier, op. cit., p. 112. Voir l'approche de certains médiévistes, B. CuRSENTE, M. Mousnier éd., Les territoires du médiéviste, Rennes, 2005. Cf. aussi, M. Bourin, E. ZadoraRio, «Analyses de l'espace» dans O. G. OexLe, J.-C. SchmitT éd., Les tendances actuelles de l'histoire du Moyen Âge en France et en Allemagne, Paris, 2002, p. 493-510. 
En invitant les chercheurs qui ont accepté de nous rejoindre, nous nous sommes posé un certain nombre de questions que nous voudrions rappeler, pour conclure, avant de leur laisser la parole.

Finalement, quelles ressources - du type que nous venons de décrire furent véritablement utilisées au Moyen Âge ? Les connaissons-nous toutes ? N'a-t-on pas privilégié l'étude de certaines ressources et laissé dans l'ombre - faute parfois de vouloir les voir ou de pouvoir les identifier - des ressources qui localement ou plus largement ont été exploitées ?

Comment les connaît-on? Quels termes les hommes du Moyen Âge utilisaient-ils pour les désigner? Quelle représentation s'en faisaient-ils ${ }^{22}$ ? Combien de temps furent-elles utilisées ? Les mille ans du Moyen Âge nous offrent - semble-t-il - un cadre de réflexion suffisamment long pour apprécier des évolutions.

Comment techniquement les ressources étaient-elles exploitées? Par quels procédés, dans quel cadre organisationnel ?

Quels espaces étaient destinés à l'exploitation de ces ressources ? Comment ces espaces, ces territoires étaient-ils dénommés, gérés ? Servaient-ils à l'exploitation de plusieurs ressources ? Quel impact sur les sociétés ou les milieux, à différentes échelles, l'exploitation des ressources eut-elle ?

De quelles façons les ressources étaient-elles contrôlées ? Leur emploi suscita-t-il des rivalités, des conflits ? À qui profitaient-elles?

Les contributions de ce numéro apportent quelques éléments de réponse à ces questions. Elles témoignent de l'ampleur et de la richesse d'un thème qui a encore peu retenu l'attention des médiévistes.

Philippe Bernard, CNRS LAMM UMR 6572, 5 rue du Château de l'Horloge, BP 647, F-13094 Aix-en-Provence Cedex 2

Didier BoIsseuIL, Université François-Rabelais, UFR Arts et Sciences humaines, Département d'Histoire, 3 rue des Tanneurs, BP 4103, F-37041 Tours Cedex

22. Indispensable selon M. GodelieR, op. cit., p. 51. Les limites de l'importance des représentations sont abordées dans G. CHOUQUER, « Nature, environnement et paysage au carrefour des théories », Études Rurales, 157-158, 2001, p. 235-252. 